\title{
On the Limits of High Angle Annular Dark Field (HAADF) Tomography; Electron Beam Damage
}

\author{
M Weyland* \\ * School of Applied \& Engineering Physics, Cornell University, Ithaca, NY 14853, USA
}

Recent research in electron tomography technique has shown several interesting trends, including the application to materials systems of bright field (BF) tomography techniques originally developed for the biosciences [1] and the use of alterative imaging modes for tomographic analysis, predominantly of materials specimens. These modes include high angle annular dark field (HAADF) scanning transmission electron microscopy (STEM) and energy filtered transmission electron microscopy (EFTEM) [2]. The fundamental limitation to biological BF tomography (in terms of resolution and fidelity) is electron beam damage [3]. However this limitation has not been explored for either the tomographic analysis of materials specimens, which are typically orders of magnitude less beam sensitive than organic specimens, or for the application of HAADF STEM tomography to biological specimens [4], where the dynamics of electron dose are very different than for BF TEM.

The effect of beam damage induced during a STEM HAADF tilt series of a biological specimen has been studied while acquiring a tilt series from a cluster of ferritin molecules in a human liver section. These molecules form hollow shells which act as storehouses for Fe, isolating it from the rest of the cell. The specimen was prepared by freeze substitution and sectioned by ultra microtome. To provide a conduction path the sample was supported on continuous carbon film and evaporation coated with a thin layer of carbon. The specimen was mounted in an ultra high tilt holder (Fischione model 2020) and examined in an fei tecnai F20 FEG-(S)TEM. Freeze substituted specimens typically undergo a rapid initial beam damage after which the specimen is relatively stable [5], to provide an even initial damage (or "clearing") the entire grid hex was evenly illuminated with a spread TEM probe for 30 minutes. A tilt series was acquired from the ferritin cluster shown in Fig. 1 a) at a STEM magnification of $320 \mathrm{kx}$ (field of view $384 \mathrm{~nm}$ ), $731024^{2}$ images were acquired from $\pm 72^{\circ}$ with a $2^{\circ}$ increment using Xplore3D (fei co.) automated acquisition software. The tilt series was aligned and reconstructed, using iterative methods, with routines coded in IDL (RSI inc.). A voxel projection from a subsection of the reconstructed volume is shown in Fig. 1 b).

A summary of the acquisition parameters and the respective dose incurred, for both the tilt series and associated automated tracking and focussing, is displayed in Table. 1. An approximate value of the probe current $(10 \mathrm{pA})$ was experimentally determined for similar conditions in a Philips CM300 FEG-(S)TEM. These figures do not account for the increase in area/volume exposed at higher tilts and therefore represents an upper bound for the total exposure. However the total calculated dose, approximately $16,000 \mathrm{eA}^{-2}$, is an order of magnitude higher than the maximum recommended for $\mathrm{BF}$ tomography [5] (typically between 40-100 $\mathrm{eA}^{-2}$ ). However the reconstruction contains fine detail, on the scale of 1-2 nm, of the partial Fe filling of some ferritin molecules, see Fig. $1 \mathrm{c}$ ) \& d). It is this high resolution detail which would be the first to be lost due to beam damage.

This result provides strong evidence that the beam damage kinetics in biological specimens during STEM is very different than for BF TEM; damage appears to be a factor not of total dose, but of the dose rate. Intriguingly these results also raise the prospect that STEM may be well suited, at least in 
terms of beam damage, for application in biological tomography.

[1] J. Frank, Electron Tomography: Three-dimensional imaging with the Transmission Electron Microscope, Plenum Press, New York; London, 1992

[2] P.A. Midgley and M. Weyland, Ultramicroscopy 96(3-4) (2003) 413

[3] B.F. McEwen, K.H. Downing and R.M. Glaeser, Ultramicroscopy 60(3) (1995) 357

[4] U. Ziese, et al., Journal Of Structural Biology 138(1-2) (2002) 58

[5] P.K. Luther, in Electron Tomography: Three-Dimensional Imaging with the Transmission Electron Microscope, J. Frank (Editor), Plenum Press, New York; London, 1992, 39

[6] The collaboration of Dr P.A.Midgley (University of Cambridge), Dr A. Brown, Dr R. Brydson (University of Leeds) and Dr A. Wharley (Kings College London) is gratefully acknowledged. Funding was provided by the Royal Commission for the Great Exhibition of 1851, EPSRC and the Isaac Newton Trust.
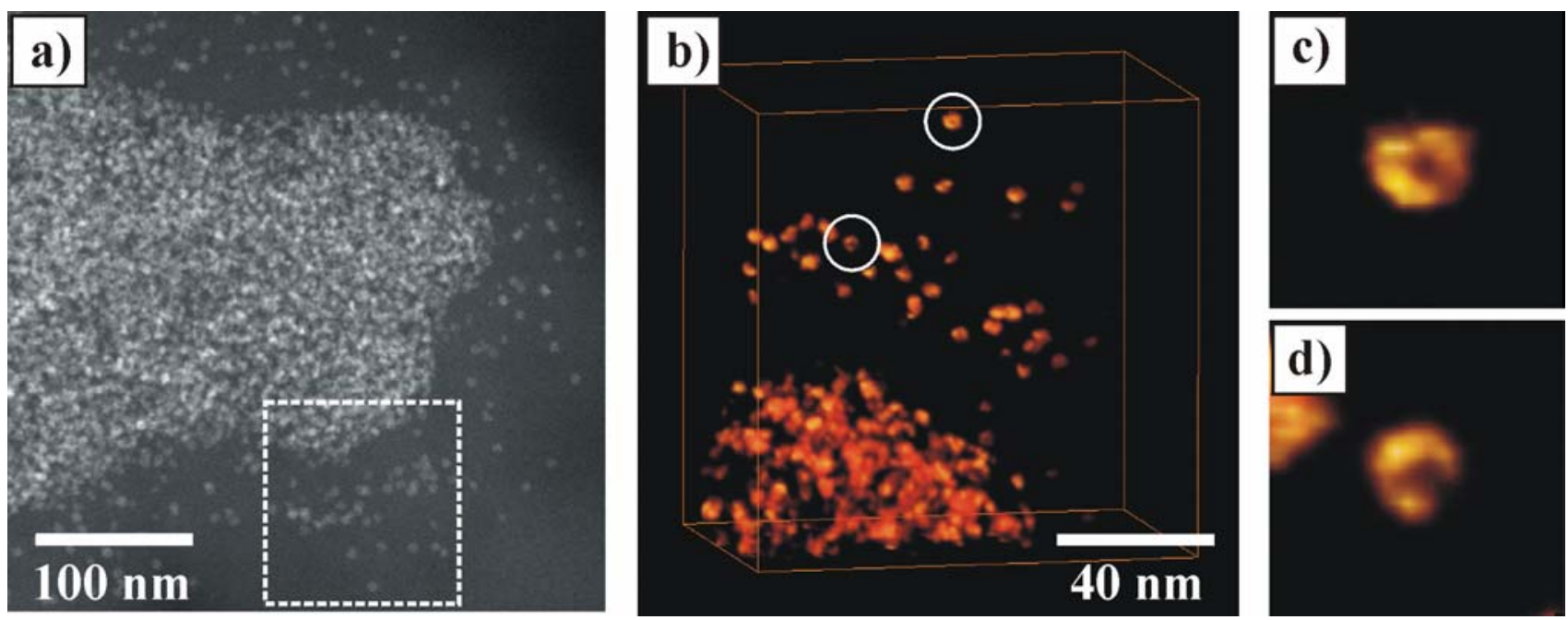

FIG 1. a) STEM HAADF image, at $320 \mathrm{kx}$, of clustered ferritin (small bright patches) in a human liver section. b) Voxel projection of a volume selected from the reconstruction, from the area marked in a box in a). c) and d) zoomed images of two ferritin clusters, circled in b), that show a distinct void in their centre. Each ferritin cluster is $\sim 5 \mathrm{~nm}$ in diameter.

TABLE 1. Approximate dose induced to a specimen of human liver section during an experimental STEM HAADF tilt series. Based on a probe current of $\sim 10 \mathrm{pA}$ and a field of view of $384 \mathrm{~nm}$.

\begin{tabular}{lccc}
\hline \multicolumn{1}{c}{ Acquistion details } & $\begin{array}{c}\# \text { of } \\
\text { images }\end{array}$ & $\begin{array}{c}\text { Dose per image } \\
\left(\mathrm{eA}^{-2}\right)\end{array}$ & $\begin{array}{c}\text { Dose for acquisition } \\
\left(\mathrm{eA}^{-2}\right)\end{array}$ \\
\hline $\begin{array}{l}\text { Acquired tilt series, one 1024x1024 image per tilt, } \\
32 \mu \text { per pixel }\end{array}$ & 72 & 140 & 10080 \\
$\begin{array}{l}\text { Series tracking, one 512x512 image per tilt, } 11 \mu \mathrm{s} \\
\text { per pixel }\end{array}$ & 71 & 12 & 852 \\
$\begin{array}{l}\text { Autofocusing, 6 512x512 images per tilt, } 11 \mu \mathrm{s} \text { per } \\
\text { pixel }\end{array}$ & 432 & 12 & 5184 \\
\hline
\end{tabular}

